Original Paper http://ajol.info/index.php/ijbes http://indexmedicus.afro.who.int

\title{
Caractérisation morpho-biométrique des populations bovines locales sans bosse du Togo
}

\author{
Soudah BOMA ${ }^{1,2^{*}}$, Yaovi NUTO ${ }^{2}$, Guiguigbaza-Kossigan $\mathrm{DAYO}^{3}$, Bèdibètè $\mathrm{BONFOH}^{1}$ et \\ Toï N'FEIDE ${ }^{4}$ \\ ${ }^{1}$ Institut Togolais de Recherche Agronomique (ITRA), Togolais, BP : 1163, Lomé, Togo. \\ ${ }^{2}$ Faculté des Sciences de l'Université de Lomé (FDS-UL), BP : 1515, Lomé, Togo. \\ ${ }^{3}$ Centre International de Recherche Développement sur l'Elevage en Zone Subhumide (CIRDES), \\ 01 BP : 454 Bobo-Dioulasso 01, Burkina Faso, \\ ${ }^{4}$ Centre de Recherche Agronomique du Littoral (CRAL) de l'Institut \\ Togolais de Recherche Agronomique (ITRA), BP : 2318 Lomé, Togo. \\ *Auteur correspondant, E-mail: bomasoudah@gmail.com
}

\section{RESUME}

Au Togo, il existe une population bovine locale sans bosse dont le phénotype varie avec la zone agroécologique. L'objectif de la présente étude était d'identifier les écotypes éventuels sur la base des traits descriptifs fournis par les propriétaires de troupeaux et des mesures morpho-biométriques des animaux. De février 2015 à avril 2016, une enquête prospective a inclus 609 vaches et 48 taureaux. Les bovins échantillons ont été pris aléatoirement dans quatre zones agro-écologiques : savane sèche, savane humide, forêt et littoral. Les analyses multi-variées et discriminantes ont porté sur le type de cornage et sur les mensurations corporelles suivantes : longueur du corps, tour de poitrine, hauteur au garrot, profondeur du thorax, longueur de la queue, longueur du crâne, largeur du front, tour du mufle, longueur des cornes et la longueur des oreilles. Une variabilité des cornages, en trois types principaux, a été observée avec un effet significatif de l'âge des animaux. L'analyse quantitative a révélé également trois types de bovins corrélés aux noms locaux donnés par les éleveurs. Les résultats de la présente étude doivent être confirmés par une caractérisation par des marqueurs moléculaires pour mieux définir la diversité génétique des populations bovines locales sans bosse du Togo et déterminer d'éventuelles structurations en « races » ou « écotypes » distincts.

(C) 2018 International Formulae Group. All rights reserved.

Mots clés : Bovins sans bosse, caractérisation, cornages, zone agroécologique, Togo.

\section{Morpho-biometric characterization of local humpless cattle populations of Togo}

\begin{abstract}
In Togo, there is a local humpless cattle population phenotypically different with the agro-ecological area. The objective of the present study was to identify eventual ecotypes of cattle based on the physical characteristics provided by the breed keepers and the morpho-biometric measurement data. From February 2015 to April 2016, an exploratory survey has included 609 cows and 48 males. The samples included four
\end{abstract}


agro-ecological areas: the dry savannah, wet savannah, costal area and forest area. Multivariate and discriminating statistics analysis were used to describe the horn types and morpho-biometric traits: body length, chest circumference, height at withers, length of ears, skull length, muzzle circumference, tail length, thorax depth, horn length and forehead width. A variability of the horn orientation was observed and revealed three principal types, a significant effect of the age of animals. The discriminating analysis of information collected from the breeders revealed three subpopulations, in correlated with the local names. The present results have to be confirmed with molecular characterization studies to define the genetic diversity and structure of local humpless cattle and confirm possible structurations into «breeds » or « ecotypes ».

(C) 2018 International Formulae Group. All rights reserved.

Keywords: Local Humpless cattle, characterization, horn types; agroecological area, Togo.

\section{INTRODUCTION}

La grande sécheresse des années 1970 a conduit à un bouleversement dans la répartition des races bovines d'Afrique de l'Ouest. Avant cette période, deux principales espèces de bovidés domestiques coexistaient : les taurins ou bovins sans bosse cervicothoracique (Bos taurus) et les zébus (bovins avec une bosse cervico-thoracique ou une bosse thoracique, Bos indicus) (MoazamiGoudarzi et al., 2001). Au nord, la latitude $12^{\circ}$ constituait la limite sud de l'aire d'élevage du Zébu ; la bande des pays côtiers étant la zone traditionnelle d'élevage des taurins à courtes cornes.

Au Togo, le nom «bovin local» désigne la population bovine Somba dans les zones agro-écologiques de la savane sèche et la savane humide (Adanléhoussi et al., 2003 ; Lombo, 2002), la population bovine Lagunaire, la population bovine Borgou et les produits très peu stabilisés de leurs croisements avec les zébus (zébus peuls transhumants) et les N'Dama, notamment. Ce sont des animaux de petite taille au garrot entre 0,96 et 1,15 mètre (Adanléhoussi et al., 2003), élevés en petits troupeaux et bien adaptés à leur milieu naturel d'élevage (Youssao et al., 2013; Talaki et al., 2014 ; Chabi China et al., 2016). Les données chiffrées sur ce cheptel sont anciennes et disparates. Aussi, seule la race bovine Somba a été caractérisée sur le plan phénotypique (Adanléhoussi et al., 2003) et moléculaire (Moazami-Goudarzi et al., 2001) dans son berceau au nord-est du Togo.

La chaîne de l'Atakora qui prend en écharpe le Togo isole au nord les sites naturels d'élevage de la savane sèche, où se trouve le berceau de la race Somba et au sud les élevages de la plaine de la savane humide. Dans la partie sud du pays, le bovin local de type Somba rencontré, se distinguerait de celui de la savane sèche du nord. En effet, les animaux du sud ont un grand gabarit, une bonne conformation par rapport à ceux de la savane sèche du nord et portent des noms locaux différents (Adomefa et Bonfoh, 2003 ; ITRA, 2005) à savoir : bovin Tém, bovin de Kouka et bovin Métis. Toutes ces observations ne reflètent pas la diversité phénotypique des populations bovines sans bosse du Togo car elles sont issues d'études parcellaires. Des études approfondies couvrant toute l'étendue du territoire sont nécessaires pour clarifier le statut du bovin local sans bosse du Togo en intégrant les données de mesures phénotypiques et les connaissances locales détenues par les éleveurs et propriétaires de ces animaux.

L'objectif de la présente étude était d'identifier les écotypes éventuels sur la base des traits descriptifs fournis par les propriétaires des troupeaux et des mesures morpho-biométriques des animaux.

\section{MATERIEL ET METHODES \\ Caractéristiques agro-écologiques des zones d'enquête}

L'enquête a concerné les quatre zones agro-écologiques du Togo, du nord au sud : la savane sèche (ZSS), la savane humide (ZSH), la zone des montagnes et plateaux ou zone de la forêt (ZF) et la zone du littoral (ZL) (Figure 1). La moyenne pluviométrique sur l'ensemble des quatre zones est d'environ 
$1250 \mathrm{~mm}$ avec un minimum de $1000 \mathrm{~mm}$ en ZSS et un maximum de $1600 \mathrm{~mm}$ en ZL. Elle est de $1300 \mathrm{~mm}$ dans la ZSH et la ZF. Géographiquement, la chaîne montagneuse de l'Atakora (sous-zone montagneuse de l'Atakora) sépare la ZSS de la ZSH et isole les sites d'élevage de Djarkpanga (Plaine du Mô) des sites de Sokodé.

\section{Collecte des données}

L'étude a porté sur 609 vaches âgées de 4 à 20 ans et de 48 mâles âgés de 3 à 10 ans dans cinq sites (Bombouaka, Kéran-Kara, kouka-Djarkpanga, Tchaoudjo et ForêtLittoral). Ces sites d'élevage correspondent respectivement aux noms locaux de bovins suivants: Mango, Somba, Kouka, Tém et Métis. L'âge des animaux de l'échantillon a été fourni par les bouviers sur la base du nombre de vêlages.

Les effectifs des animaux échantillonnés par site et par nom local du bovin sont reportés dans le Tableau 1 .

En février 2015, une enquête prospective a été conduite auprès des éleveurs organisés en groupes dans les villages et deux villages ont été sélectionnés par site. L'interview semi-direct pour la collecte de données qualitatives a été administré et a porté sur les directives suivantes : le nom local des bovins types, leurs caractéristiques particulières, leurs origines probables et leur zone d'extension géographique.

L'enquête transversale pour la collecte des données morpho-biométriques sur les animaux s'est déroulée de mars à mai en 2015 puis de mars à avril 2016. En absence des données chiffrées sur l'effectif total du cheptel dans la zone de l'étude et des informations précises sur la position géographique des troupeaux, l'approche aléatoire, sans quotas d'échantillon (le troupeau puis le bovin) a été adoptée.

Les caractéristiques quantitatives ont été mesurées en centimètre et ont porté sur : la longueur du corps (LC), le tour de poitrine (TP), la hauteur au garrot (HG), la profondeur du thorax (PTh), la longueur de la queue
(LQ), la longueur du crâne (LCr : de la nuque à l'incision du mufle), la largeur du front (LF), le tour du mufle (TM), la longueur des cornes (LCn) et la longueur des oreilles (LO) (Figure 2).

Pour chaque animal, les photos ont été prises pour décrire le type de cornage. La Figure 3 (photos $\mathrm{A}$ à photo $\mathrm{J}$ ) illustre les dix patrons de cornages utilisés pour décrire les animaux à savoir :

- la forme coupe dont la coupe rectiligne concave (Cp-RC) et la coupe évasée (Cp-E) ;

- la forme croissant comprenant le croissant rectiligne (Cr-R), le croissant incurvé au stade juvénile (Cr-IJ), le croissant incurvé au stade avancé (Cr-IA) et le croissant rabattu avant (Cr-RAv);

- le type ligne dont la ligne rectiligne $(\mathrm{L}-\mathrm{Rec})$ et la ligne rabattue arrière (L- RAr) ;

- la forme asymétrique dont le type déformée gauche (ADG) et le type déformé droit (ADD) ;

- le type sans corne, le type roue, le type spiral extérieur, le type moignon (cornes courtes ne dépassant pas $5 \mathrm{~cm}$ de longueur) et le type flottant.

\section{Analyses statistiques}

Le logiciel SPSS 20 a été utilisé pour les analyses statistiques. Les effectifs et les fréquences décrivant le type de cornage ont été évalués. Pour les variables quantitatives, les moyennes, écart-types et valeurs extrêmes ont été calculés pour chaque zone agroécologique.

Le test Post Hoc de Tukey a été utilisé, séparément pour les variables qualitatives et les variables quantitatives et la classification du plus proche voisin $(\mathrm{KNN})$, pour ressortir les sous-ensembles homogènes. La procédure GLM (Modèle Linéaire Généralisé) basée sur la distance de Mahalanobis (D-Mahalanobis) a été utilisée pour identifier les variables permettant de discriminer au mieux les écotypes et ainsi, valider la classification. 


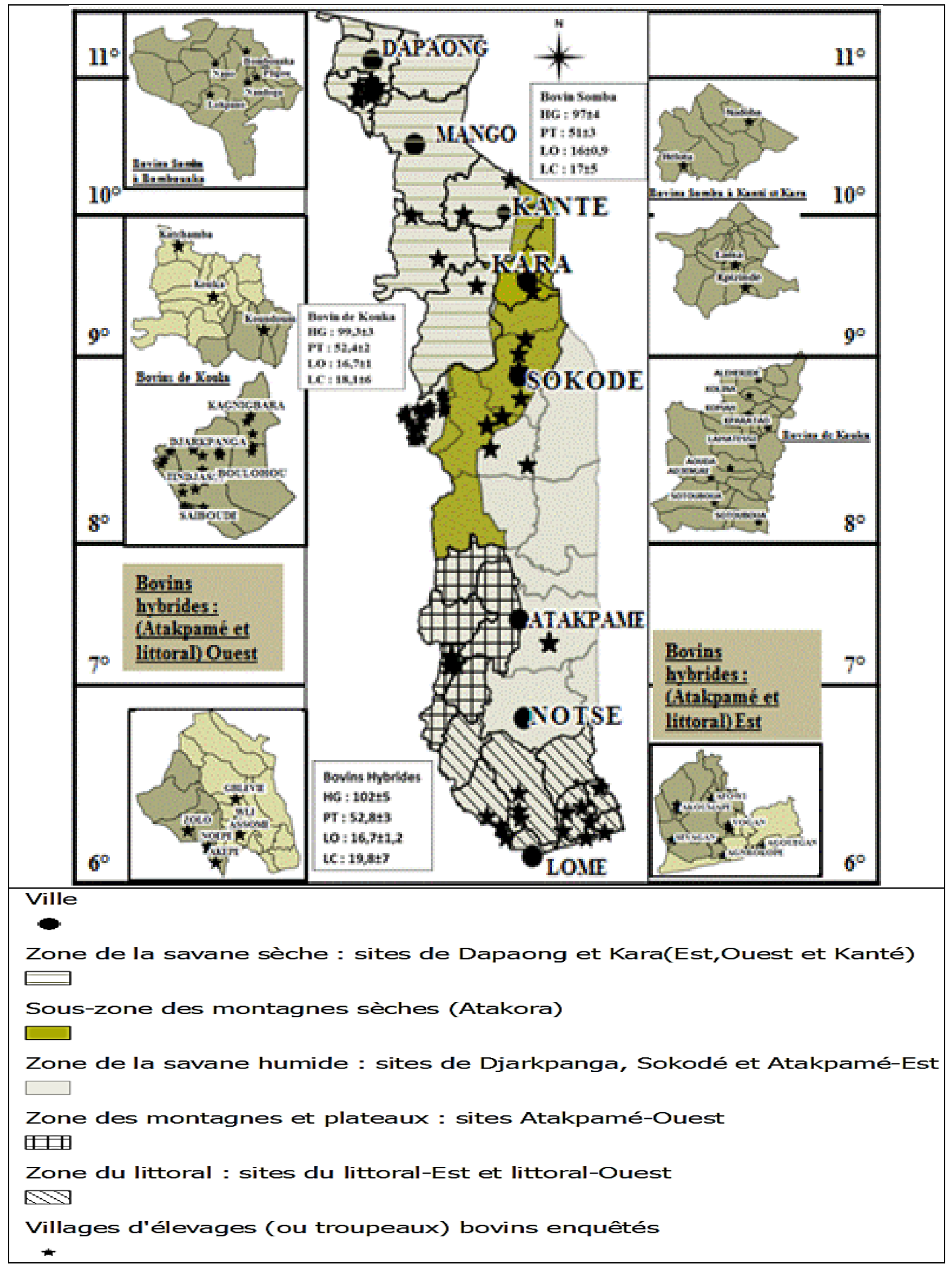

Figure 1 : Localisation des sites d'enquête et répartition de types du bovin local. 
Tableau 1 : Effectifs des vaches et des taureaux échantillonnés par site.

\begin{tabular}{lccccc}
\hline Sites d'enquête & $\begin{array}{c}\text { Superficie } \\
\left(\mathbf{e n ~ k m}^{\mathbf{2}}\right)\end{array}$ & Nom local du bovin & Vaches & Taureaux & Total \\
\hline Bombouaka & 95,5 & Bovin de Mango & 59 & 08 & 67 \\
Kéran-Kara & 310,5 & Somba & 99 & 18 & 117 \\
Kouka-Djarkpanga & 379,9 & Bovin de Kouka & 181 & 06 & 187 \\
Tchaoudjo & 554,3 & Bovin Tém & 168 & 09 & 177 \\
Forêt-Littoral & 752,3 & Métis (Hybride) & 102 & 07 & 109 \\
Total & & & $\mathbf{6 0 9}$ & $\mathbf{4 8}$ & $\mathbf{6 5 7}$ \\
\hline
\end{tabular}
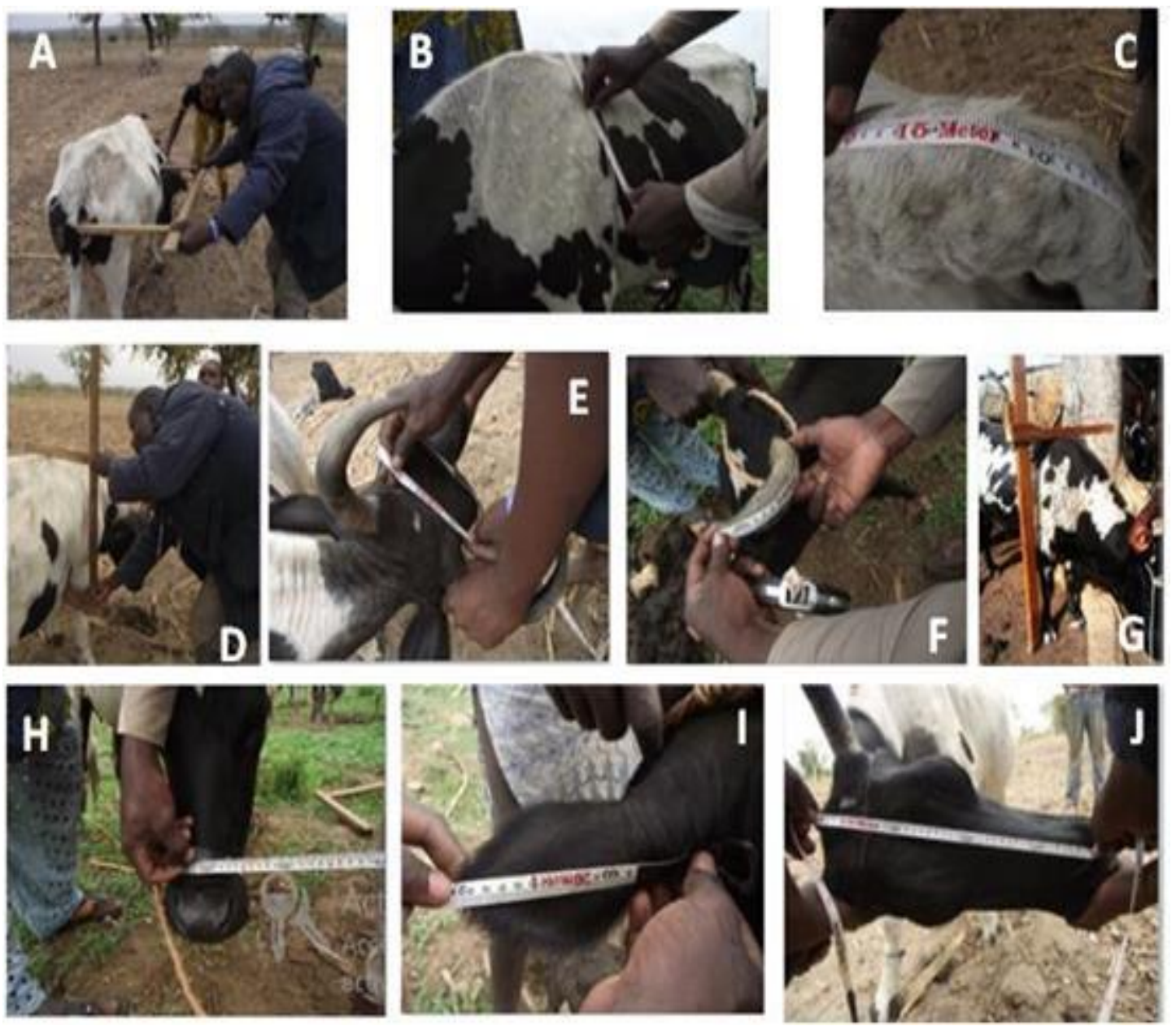

A : la longueur du corps, B : tour de poitrine, $\mathrm{C}$ : la longueur de la queue, D : la profondeur du thorax, E : la largeur du front, F : la longueur des cornes, $\mathrm{G}$ : hauteur au garrot, $\mathrm{H}$ : le tour du mufle, I : la longueur des oreilles, $\mathrm{J}:$ la longueur du crane (de la nuque à l'incision du mufle).

Figure 2: Illustration de quelques mensurations réalisées. 


\section{RESULTATS Caractéristiques cornage \\ morphologiques de}

Les fréquences des différentes formes de cornage selon les sites sont résumées dans le Tableau 2. L'analyse de l'effet de l'âge a révélé que le type Cr-IA est associé à un âge avancé (âge $\geq 10$ ans), tandis que le type coupe rectiligne concave est associé à un âge jeune (âge compris entre 4 et 5 ans) (Tableau 3). La comparaison des fréquences des cornages observés a révélé également une variabilité (coefficient de contingence : 0,53; Phi : 0,63; V de Cramer : 0, 31) selon deux principaux groupes.

Dans la zone du centre et du nord, de Sokodé à Dapaong, un peu plus de la moitié (50,4\% sur 507 dans les deux zones) des vaches ont un cornage de type croissant, avec une dominance de la variante Cr-IJ (114 sur 254 observations, soit 44,8\%) suivi du cornage de type coupe avec $36,6 \%$ de l'échantillon. Toutefois, le site naturel des bovins Somba (Kanté-Est ou Nadoba) se singularise par une forte proportion $(55,5 \%$ de l'échantillon) du type Coupe suivi du type Croissant $(41,4 \%)$. En revanche, sur le site de Tchaoudjo, le type croissant incurvé est assez important $(46,4 \%)$. Ce type est présent également dans les autres populations bovines : 26,4\% à Kanté, $33 \%$ à Kouka-Djarkpanga et $38,9 \%$ à Bombouaka, montrant qu'il constitue également un trait commun pour l'ensemble des animaux de la zone nord du pays.

Dans la zone sud, du littoral jusqu'à la limite d'Atakpamé (zone agro-écologique de la forêt), la population bovine hybride (métisse ou produits de divers croisements) est caractérisée par un cornage de type croissant rectiligne (Figure 3: photo B1): $42,2 \%$ de l'échantillon de la zone. Dans cette zone agro-écologique, le cornage en coupe a représenté $24,5 \%$, suivi du type en Lyre $(13,7 \%)$. Ce groupe se distingue également par la présence du type sans cornes ( 3 bovins sur 102) et l'absence des types moignons, roue et spiral.

\section{Traits quantitatifs et analyse multivariée}

Les résultats morpho-biométriques obtenus montrent globalement un gradient décroissant des mensurations quantitatives du sud vers le nord (Tableau 4). L'effet du site d'enquête a été très significatif $\left(\mathrm{R}^{2}>25 \%\right)$ pour six variables (HG, PTh, LC, TP, LO et $\mathrm{LCn})$ et moins significatif $\left(\mathrm{R}^{2}<25 \%\right)$ pour quatre variables (LF, $\mathrm{LCr}, \mathrm{TM}$ et LQ) (Tableau 5). L'analyse basée sur la classification du plus proche voisin a révélé que les variables pour lesquelles l'effet statistique du site d'enquête est très significatif (Figure 4) sont celles qui discriminent le mieux ces sites en sousensembles homogènes.

Les résultats des analyses multivariées ont donné la même tendance sur le nombre de populations bovines identifiées par les éleveurs en distinguant trois sous-groupes homogènes. Ces trois sous-groupes correspondent à la répartition des fréquences des types de cornages.

L'analyse discriminante canonique basée sur les effectifs par zone agroécologique a révélé que deux principaux facteurs permettent de typer les trois populations bovines de l'étude. Le premier facteur cumule la quasi-totalité $(92,6 \%)$ de la variance expliquée par cinq variables (TP, HG, PTh, LCn et LO) qui caractérisent mieux les trois groupes. Le second facteur contribue très peu à l'inertie du tableau des variables et est lié à la variable TM. Sur la base de ces deux principaux facteurs de classification, une proportion satisfaisante de $75,9 \%$ des observations a été classée. Toutefois, une proportion assez importante $(62,7 \%)$ des bovins de la zone agro-écologique de la savane sèche (sites de Kanté et Mango) ont été considérés comme bovins de Kouka. L'analyse détaillée des classes d'affectation montre que $71,7 \%$ de ceux-ci sont hors des sites naturels (Kéran-Est) d'élevage du bovin Somba. L'analyse uni-variée appliquée au carré de la distance de Mahalanobis au barycentre (Tableau 6) a révélé que, globalement les trois populations se distinguent statistiquement (Moyenne des carrées intergroupes : 60,1; F : 8,1). Le test de Tukey de l'analyse discriminante montre une proximité, non très étroite, entre la population bovine de Kouka (D-Mahalanobis : 1,5) et celle des Hybrides confirmés (D- 
Mahalanobis: 2,1). Ces deux groupes se distinguent statistiquement des Somba vrais ou bovins de la zone naturelle d'élevage des Somba (D-Mahalanobis : 3,1).

\section{Caractéristiques morpho-biométriques des populations bovines identifiées}

Les caractéristiques morphobiométriques des animaux ont permis de distinguer trois populations bovines locales sans bosse (Figure 4) :

(i) Plus au sud, les hybrides métis confirmés par les éleveurs, lourds, caractérisés par les valeurs maximales des mesures: PTh, LC et TP. Ils sont hauts sur pattes (HG : 107 $\mathrm{cm}$ ) avec des oreilles (en moyenne $18 \mathrm{~cm}$ ) et des cornes (en moyenne $29 \mathrm{~cm}$ ) relativement longues. Les autres mesures se présentent comme suite : PTh : 57,2 $\pm 2,5 \mathrm{~cm}, \mathrm{LC}: 117,9$ $\pm 6,2 \mathrm{~cm}, \mathrm{TP}: 147,2 \pm 7,1 \mathrm{~cm}, \mathrm{LF}: 15,2 \pm 0,7$ $\mathrm{cm}, \mathrm{LCr}: 39,8 \pm 1,8 \mathrm{~cm}, \mathrm{LQ}: 84,8 \pm 6,5 \mathrm{~cm}$ et TM: $36,9 \pm 1,9 \mathrm{~cm}$. Leur cornage est à dominance de type croissant rectiligne.

(ii) Au Nord, de la zone des plateaux et cuesta à la zone des montagnes sèches, la population bovine Somba est caractérisée par des individus courts sur pattes (HG : 97,4 \pm
4,5 cm), relativement moins lourds (TP : 129,1 \pm 6,7 cm, PTh : 50,8 $\pm 3,4 \mathrm{~cm}$ et LC : $108,1 \pm 5,4 \mathrm{~cm})$ aux oreilles courtes $(15,9 \pm$ $0,9 \mathrm{~cm}$ ). Les moyennes des autres mesurent se présentent comme suit : $\mathrm{LCn}: 17,6 \pm 5,7 \mathrm{~cm}$, LC : $14,8 \pm 0,7 \mathrm{~cm}, \mathrm{LCr}: 37,4 \pm 2 \mathrm{~cm}, \mathrm{TM}$ : $34,81,8 \mathrm{~cm}$ et LQ : $76,4 \pm 5,6 \mathrm{~cm}$. Le cornage fréquemment rencontré est le type coupe, associé souvent au type croissant incurvé au stade juvénile.

(iii) Dans la zone tampon qui comprend l'ouest de la Région de la Kara et la Région Centrale, l'étude distingue une population bovine intermédiaire entre les deux précédents groupes (Figure 5). Elle se singularise sur les paramètres suivants : HG : $99,3 \pm 3,9 \mathrm{~cm}$, PTh : 52,4 $\pm 2,2 \mathrm{~cm}, \mathrm{LC}:$ $109,5 \pm 5,1 \mathrm{~cm}$, TP $: 131 \pm 6,3 \mathrm{~cm}, \mathrm{LO}: 16,7$ $\pm 1 \mathrm{~cm}, \mathrm{LC}: 18,1 \pm 6,3 \mathrm{~cm}, \mathrm{LF}: 14,9 \pm 0,9$ $\mathrm{cm}, \mathrm{LCr}: 38,2 \pm 1,5 \mathrm{~cm}, \mathrm{TM}: 35,6 \pm 1,7 \mathrm{~cm}$, et LQ : $77,9 \pm 4,9 \mathrm{~cm}$. Le cornage fréquemment rencontré est le type coupe $(58,5 \%)$. Particulièrement, le type croissant incurvé au stade avancé (26\%) est aussi rencontré alors que sur le site naturel d'élevage des Somba le pourcentage du type coupe est de $11 \%$.

Tableau 2 : Fréquences des types de cornages selon le nom local ou site d'étude.

\begin{tabular}{llcccccc}
\hline Type de cornage & & Kouka & Somba & Mango & Métisse & Tém & Total \\
\hline Cp-E & EF & $33^{\mathrm{c}}$ & $27^{\mathrm{b}}$ & $7^{\mathrm{c}}$ & $10^{\mathrm{a}}$ & $29^{\mathrm{c}}$ & 106 \\
& $\mathrm{~F}$ & $(18,2)$ & $(27,3)$ & $(11,9)$ & $(9,8)$ & $(17,3)$ & $(17,4)$ \\
$\mathrm{Cr}-\mathrm{IA}$ & $\mathrm{EF}$ & $13^{\mathrm{a}}$ & $11^{\mathrm{c}}$ & $6^{\mathrm{c}}$ & $4^{\mathrm{a}}$ & $33^{\mathrm{b}}$ & 67 \\
& $\mathrm{~F}$ & $(7,2)$ & $(11,1)$ & $(10,2)$ & $(3,9)$ & $(19,6)$ & $(11,0)$ \\
$\mathrm{Cr}-\mathrm{IJ}$ & $\mathrm{EF}$ & $47^{\mathrm{a}}$ & $15^{\mathrm{c}}$ & $17^{\mathrm{a}}$ & $8^{\mathrm{b}}$ & $35^{\mathrm{a}}$ & 122 \\
& $\mathrm{~F}$ & $(26)$ & $(15,2)$ & $(28,8)$ & $(7,8)$ & $(20,8)$ & $(19,9)$ \\
$\mathrm{Cr}-\mathrm{R}$ & $\mathrm{EF}$ & $11^{\mathrm{a}}$ & $14^{\mathrm{a}}$ & $6^{\mathrm{a}}$ & $43^{\mathrm{b}}$ & $23^{\mathrm{a}}$ & 99 \\
& $\mathrm{~F}$ & $(6,1)$ & $(16,2)$ & $(10,2)$ & $(42,2)$ & $(13,7)$ & $(16,3)$ \\
Flottant & $\mathrm{EF}$ & $2^{\mathrm{a}}$ & $0^{\mathrm{a}}$ & $0^{\mathrm{a}}$ & $3^{\mathrm{a}}$ & $1^{\mathrm{a}}$ & 6 \\
& $\mathrm{~F}$ & $(1,1)$ & $(0,0)$ & $0,0)$ & $(2,9)$ & $(0,6)$ & $(1,0)$ \\
Lyre & $\mathrm{EF}$ & $0 \mathrm{a}$ & $0 \mathrm{a}$ & $0 \mathrm{a}$ & $14 \mathrm{~b}$ & $1 \mathrm{a}$ & 15 \\
& $\mathrm{~F}$ & $(0,0)$ & $(0,0)$ & $(0,0)$ & $(13,7)$ & $(0,6)$ & $(2,5)$ \\
Moignons & $\mathrm{EF}$ & $11^{\mathrm{c}}$ & $1^{\mathrm{c}}$ & $5^{\mathrm{b}}$ & $0^{\mathrm{a}}$ & $4^{\mathrm{c}}$ & 21 \\
& $\mathrm{~F}$ & $(6,1)$ & $(1,0)$ & $(8,5)$ & $(0,0)$ & $(2,4)$ & $(3,4)$ \\
\hline
\end{tabular}

$\mathrm{EF}$ : effectif de bovin présentant le caractère ; $\mathrm{F}(\ldots)$ : fréquence de bovins présentant le caractère par rapport à l'échantillon total. Pour chaque type de cornage, les chiffres portant la même lettre en exposant indiquent que les fréquences enregistrées sur ces sites sont similaires au seuil de 5\%. Coefficient de contingence : 0,53; Phi : 0,63 et V de Cramer : 0, 31 au seuil de $5 \%$. 
Tableau 3 : Caractéristiques de cornage par catégorie d'âge de bovins local.

\begin{tabular}{|c|c|c|c|c|c|}
\hline & & âge $\geq 10$ ans & 6 - 9 ans inclus & Age $\leq 5$ ans & Total \\
\hline \multirow[t]{2}{*}{$\mathrm{ADD}$} & $\mathrm{EF}$ & $1_{\mathrm{a}}$ & $5 \mathrm{a}$ & $1_{\mathrm{a}}$ & 7 \\
\hline & $\mathrm{F}$ & $(0,8)$ & $(1,4)$ & $(0,8)$ & $(1,1)$ \\
\hline \multirow[t]{2}{*}{ ADG } & $\mathrm{EF}$ & $2 \mathrm{a}$ & $4 a$ & $0_{\mathrm{a}}$ & 6 \\
\hline & $\mathrm{F}$ & $(1,6)$ & $(1,1)$ & $(0,0)$ & $(1,0)$ \\
\hline \multirow[t]{2}{*}{ Cp-E } & $\mathrm{EF}$ & $21_{\mathrm{a}}$ & $68 \mathrm{a}$ & $18 \mathrm{a}$ & 107 \\
\hline & $\mathrm{F}$ & $(16,5)$ & $(18,8)$ & $(14,9)$ & $(17,6)$ \\
\hline \multirow[t]{2}{*}{$\overline{C p-R C}$} & $\mathrm{EF}$ & $13_{\mathrm{a}}$ & $54_{a}$ & $34_{b}$ & 101 \\
\hline & $\mathrm{F}$ & $(10,2)$ & $(15,0)$ & $(28,1)$ & $(16,6)$ \\
\hline \multirow[t]{2}{*}{ Cr-IA } & $\mathrm{EF}$ & $24_{a}$ & $37_{\mathrm{b}}$ & $6_{b}$ & 67 \\
\hline & $\mathrm{F}$ & $(18,9)$ & $(10,2)$ & $(5,0)$ & $(11,0)$ \\
\hline \multirow[t]{2}{*}{ Cr-IJ } & $\mathrm{EF}$ & $30_{a}$ & $75_{\mathrm{a}}$ & $17_{\mathrm{a}}$ & 122 \\
\hline & $\mathrm{F}$ & $(23,6)$ & $(20,8)$ & $(14,0)$ & $(20,0)$ \\
\hline \multirow[t]{2}{*}{ Cr-RAv } & $\mathrm{EF}$ & $6_{a}$ & $15_{\mathrm{a}}$ & $2 \mathrm{a}$ & 23 \\
\hline & $\mathrm{F}$ & $(4,7)$ & $(4,2)$ & $(1,7)$ & $(3,8)$ \\
\hline \multirow[t]{2}{*}{ Cr-Rec } & $\mathrm{EF}$ & $16_{a}$ & $57_{\mathrm{a}}$ & $24_{a}$ & 97 \\
\hline & $\mathrm{F}$ & $(12,6)$ & $(15,8)$ & $(19,8)$ & $(15,9)$ \\
\hline \multirow[t]{2}{*}{ Flottant } & $\mathrm{EF}$ & $0_{\mathrm{a}}$ & $4 a$ & $2 \mathrm{a}$ & 6 \\
\hline & $\mathrm{F}$ & $(0,0)$ & $(1,1)$ & $(1,7)$ & $(1,0)$ \\
\hline \multirow[t]{2}{*}{ L-RAr } & $\mathrm{EF}$ & $1_{\mathrm{a}}$ & $5 \mathrm{a}$ & $1_{\mathrm{a}}$ & 7 \\
\hline & $\mathrm{F}$ &, $8 \%$ & $(1,4)$ & $(0,8)$ & $(1,1)$ \\
\hline \multirow[t]{2}{*}{ L-Rec } & $\mathrm{EF}$ & $2 \mathrm{a}$ & $7 \mathrm{a}$ & $4_{\mathrm{a}}$ & 13 \\
\hline & $\mathrm{F}$ & $(1,6)$ & $(1,9)$ & $(3,3)$ & $(2,1)$ \\
\hline \multirow[t]{2}{*}{ Lyre } & $\mathrm{EF}$ & $4 a$ & $6 a$ & $5_{a}$ & 15 \\
\hline & $\mathrm{F}$ & $(3,1)$ & $(1,7)$ & $(4,1)$ & $(2,5)$ \\
\hline \multirow[t]{2}{*}{ Moignons } & $\mathrm{EF}$ & $2 \mathrm{a}$ & $15_{\mathrm{a}}$ & $4 a$ & 21 \\
\hline & $\mathrm{F}$ & $(1,6)$ & $(4,2)$ & $(3,3)$ & $(3,4)$ \\
\hline \multirow[t]{2}{*}{ Roue } & $\mathrm{EF}$ & $44_{a}$ & $6_{a}$ & $2 \mathrm{a}$ & 12 \\
\hline & $\mathrm{F}$ & $(3,1)$ & $(1,7)$ & $(1,7)$ & $(2,0)$ \\
\hline \multirow[t]{2}{*}{ Sans cornes } & $\mathrm{EF}$ & $0_{a}$ & $22_{a}$ & $1_{\mathrm{a}}$ & 3 \\
\hline & $\mathrm{F}$ & $(0,0)$ & $(0,6)$ & $(0,8)$ & $(0,5)$ \\
\hline \multirow[t]{2}{*}{ S-Ex } & $\mathrm{EF}$ & $1_{\mathrm{a}}$ & $1_{\mathrm{a}}$ & $0_{a}$ & 2 \\
\hline & $\mathrm{F}$ & $(0,8)$ & $(0,3)$ & $(0,0)$ & $(0,3)$ \\
\hline Total & & 127 & 361 & 121 & 609 \\
\hline
\end{tabular}




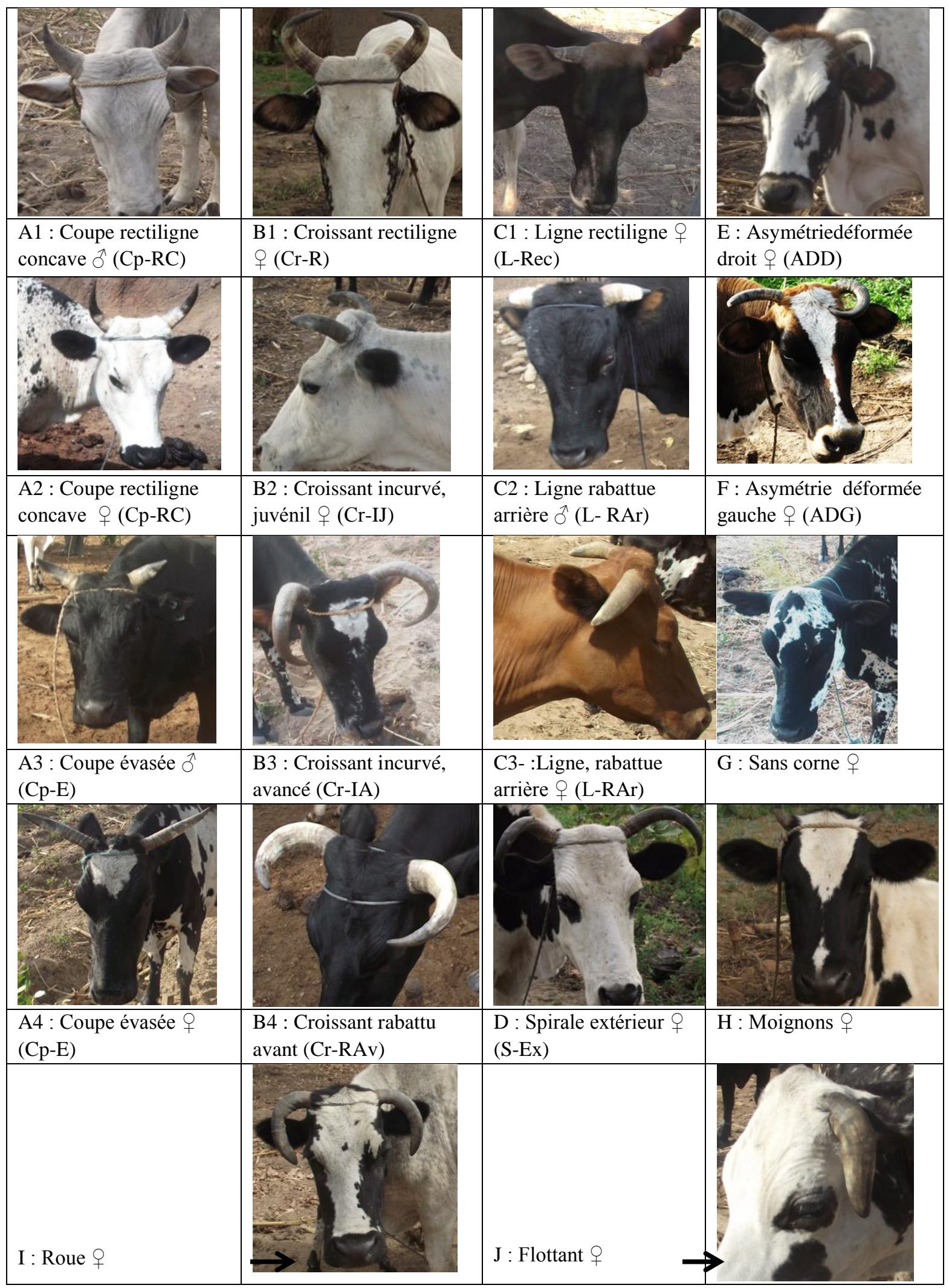

Figure 3 : Illustration des cornages du bovin local sans bosse du Togo. 
Tableau 4 : Caractéristiques des mesures selon l'appellation locale.

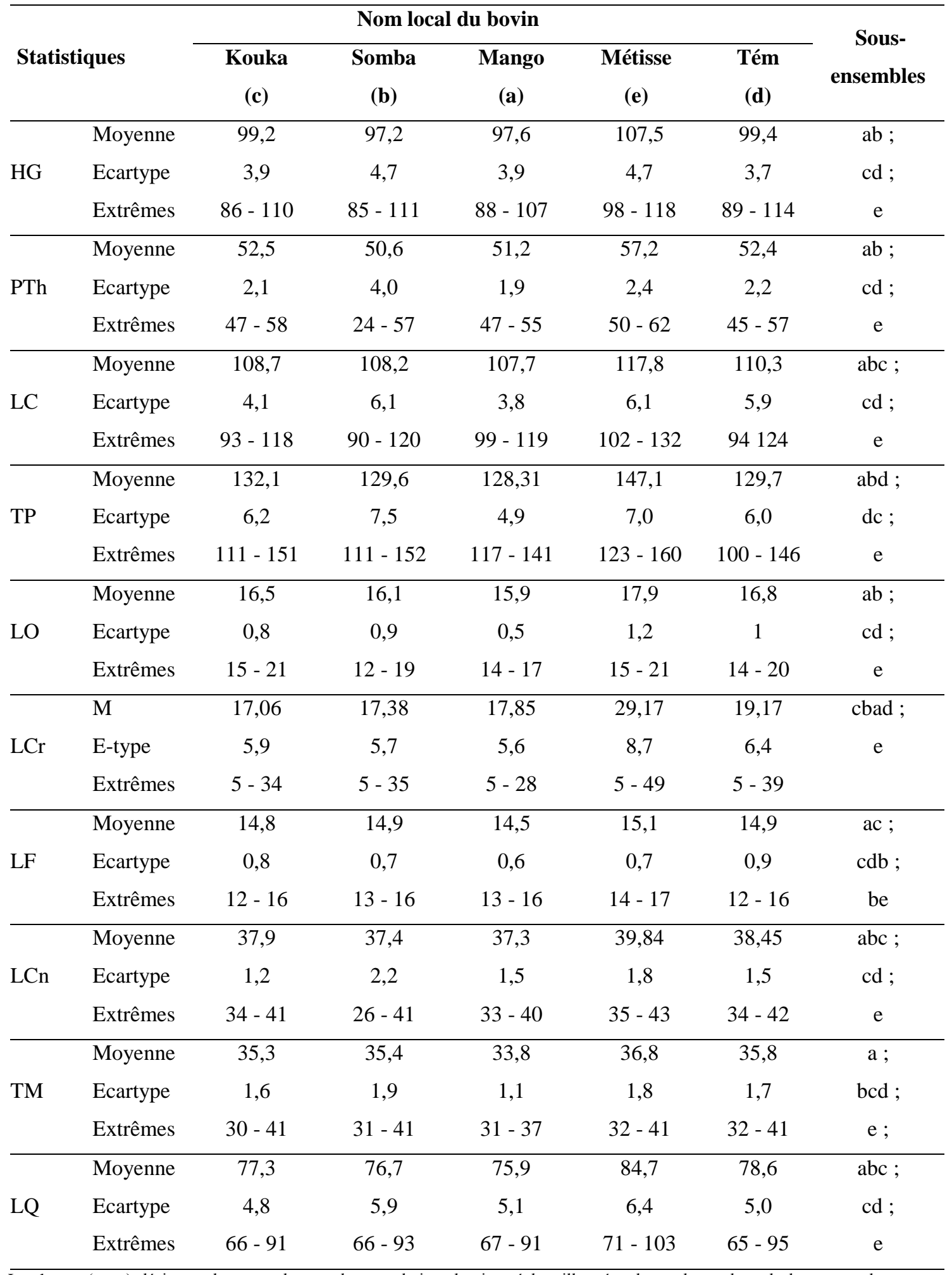

Les lettres (a...e) désignent les noms locaux des populations bovines échantillonnées du nord vers le sud, dans cet ordre. Pour chaque mesure, les sous-ensembles homogènes (Test de Tukey au seuil de 5\%) sont constitués en utilisant ces lettres. 
Tableau 5 : Statistiques multi-variées de l'effet site sur les paramètres biométriques.

\begin{tabular}{lccccc}
\hline Paramètre & Somme des carrés & ddl & Moyenne des carrés & D & Signification \\
\hline G & $7033,5 \mathrm{a}$ & 4 & 1758,4 & 99,7 & $\mathrm{~S}$ \\
PTh & $2658,6 \mathrm{~b}$ & 4 & 664,7 & 98,1 & $\mathrm{~S}$ \\
LCP & $7035,0 \mathrm{c}$ & 4 & 1758,7 & 61,5 & $\mathrm{~S}$ \\
TP & $24832,6 \mathrm{~d}$ & 4 & 6208,1 & 148,6 & $\mathrm{~S}$ \\
LO & $228,9 \mathrm{e}$ & 4 & 57,2 & 58,0 & $\mathrm{~S}$ \\
LCo & $11191,4 \mathrm{f}$ & 4 & 2797,9 & 64,6 & $\mathrm{~S}$ \\
LF & $16,0 \mathrm{~g}$ & 4 & 4,0 & 5,8 & $\mathrm{~S}$ \\
LCra & $395,4 \mathrm{~h}$ & 4 & 98,9 & 35,4 & $\mathrm{~S}$ \\
TM & $362,8 \mathrm{i}$ & 4 & 90,7 & 30,5 & $\mathrm{~S}$ \\
LQ & $4921,0 \mathrm{j}$ & 4 & 1230,2 & 42,4 & $\mathrm{~S}$ \\
\hline
\end{tabular}

$\mathrm{a}: \mathrm{R}$ deux $=0,398\left(\mathrm{R}^{2}\right.$ ajusté $\left.=0,394\right) ; \mathrm{b}: \mathrm{R}^{2}=0,394\left(\mathrm{R}^{2}\right.$ ajusté $\left.=0,390\right) ; \mathrm{c}: \mathrm{R}^{2}=0,290\left(\mathrm{R}^{2}\right.$ ajusté $\left.=0,285\right) ; \mathrm{d}: \mathrm{R}^{2}=0,496$ $\left(\mathrm{R}^{2}\right.$ ajusté $\left.=0,493\right) ; \mathrm{e}: \mathrm{R}^{2}=0,278\left(\mathrm{R}^{2}\right.$ ajusté $\left.=0,273\right) ; \mathrm{f}: \mathrm{R}^{2}=0,300\left(\mathrm{R}^{2}\right.$ ajusté $\left.=0,295\right) ; \mathrm{g}: \mathrm{R}^{2}=0,037\left(\mathrm{R}^{2}\right.$ ajusté $=$ $0,031) ; \mathrm{h}: \mathrm{R}^{2}=0,190\left(\mathrm{R}^{2}\right.$ ajusté $\left.=0,185\right) ; \mathrm{i}: \mathrm{R}^{2}=0,168\left(\mathrm{R}^{2}\right.$ ajusté $\left.=0,163\right) ; \mathrm{j}: \mathrm{R}^{2}=0,219$ ( $\mathrm{R}^{2}$ ajusté $\left.=0,214\right)$. $\mathrm{S}:$ effet statistiquement significatif au seuil de $5 \%$.

Tableau 6 : Statistiques du classement des observations selon les trois noms locaux.

\begin{tabular}{llccc}
\hline $\begin{array}{l}\text { Groupe prévu } \\
\text { (Effectif) }\end{array}$ & Statistiques & $\mathbf{P}(\mathbf{D}>\mathbf{d} \mid \mathbf{G}=\mathbf{g})$ & $\mathbf{P}(\mathbf{G}=\mathbf{g} \mid \mathbf{D}=\mathbf{d})$ & $\begin{array}{c}\text { Carré de la distance de } \\
\text { Mahalanobis au barycentre }\end{array}$ \\
\hline Somba (57) & M & 0,46 & 0,65 & 3,17 \\
& E-type & 0,30 & 0,12 & 6,89 \\
Somba** (25) & M & 0,59 & 0,57 & 1,67 \\
& E-type & 0,29 & 0,09 & 2,14 \\
Kouka (318) & M & 0,58 & 0,75 & 1,51 \\
& E-type & 0,26 & 0,12 & 1,82 \\
Kouka** (112) & M & 0,56 & 0,67 & 1,41 \\
& E-type & 0,23 & 0,11 & 1,16 \\
Hybrides (87) & M & 0,49 & 0,91 & 2,16 \\
& E-type & 0,29 & 0,12 & 2,20 \\
Hybrides** (10) & M & 0,50 & 0,76 & 1,82 \\
& E-type & 0,26 & 0,15 & 1,68 \\
Total (609) & M & 0,55 & 0,74 & 1,75 \\
& E-type & 0,27 & 0,14 & 2,74 \\
\hline
\end{tabular}

**: Observations reclassées, $\mathrm{P}(\mathrm{D}>\mathrm{d} \mid \mathrm{G}=\mathrm{g})$ : Probabilité d'appartenance au groupe de départ, $\mathrm{P}(\mathrm{G}=\mathrm{g} \mid \mathrm{D}=\mathrm{d})$ : Probabilité d'appartenance au groupe prévu par l'analyse discriminante. 


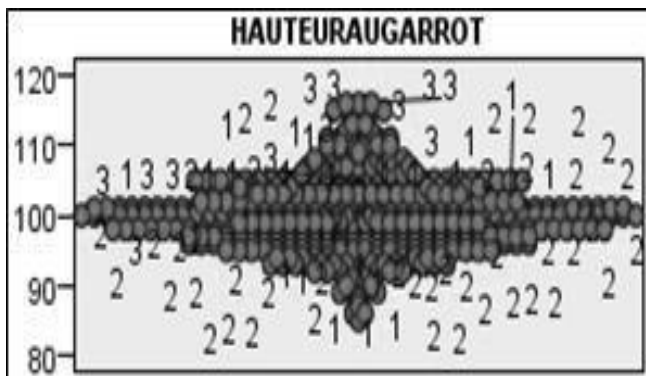

LOIIGUEURDUCORPS

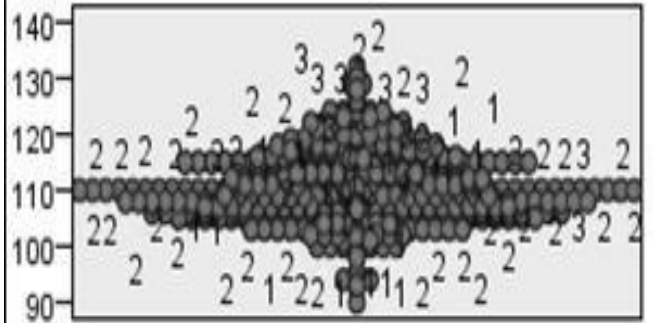

LOIIGUEURDESOREILLES

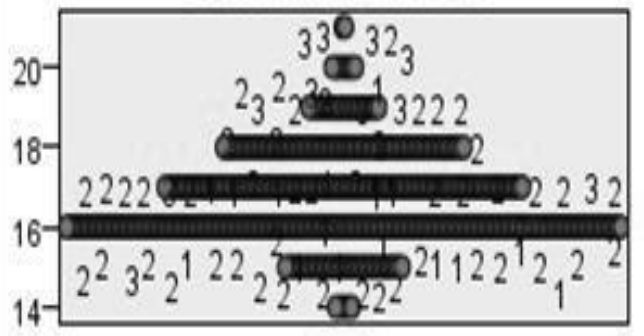

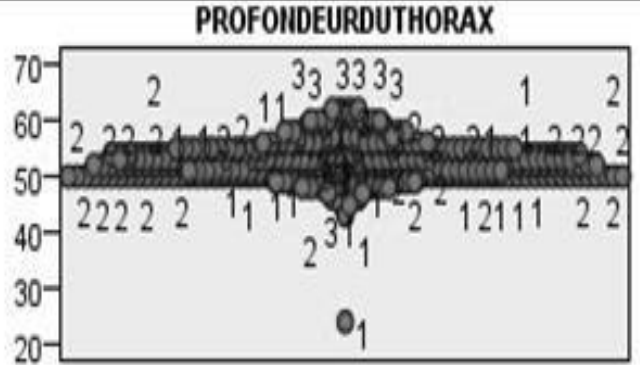

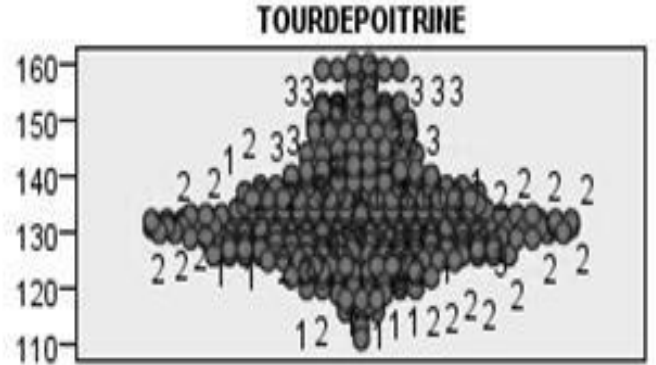

LOIIGUEURDESCORIES

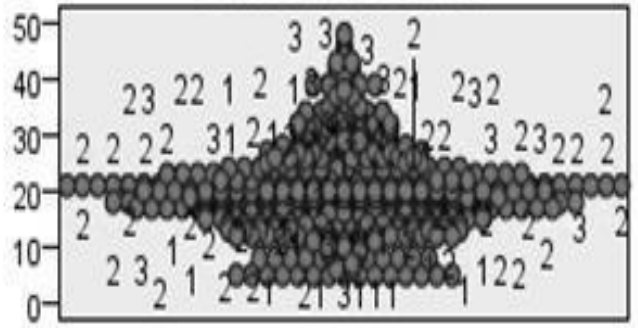

1 : Population bovine Somba ; 2 : Population bovine de Kouka ; 3 : Populations bovine hybride.

Figure 4 : Diagrammes de répartition des observations selon les mesures discriminatives.
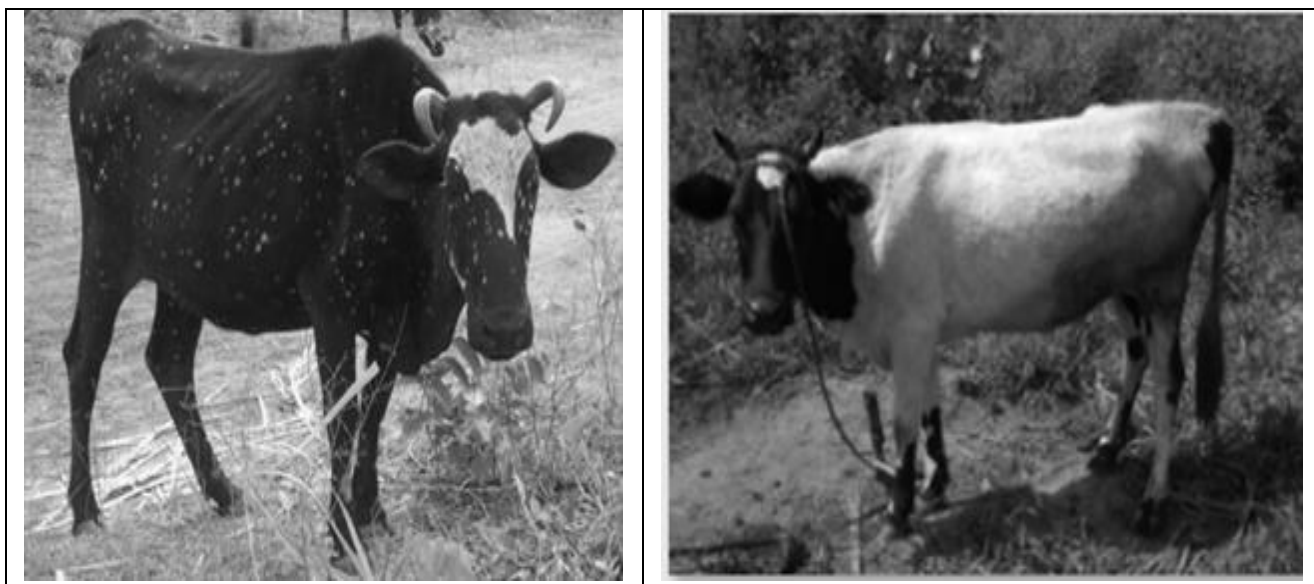

Figure 5 : Spécimens type de vaches de Kouka et de la zone Tchaoudjo (Sokodé). 


\section{DISCUSSION}

Les groupes de discussions, les observations faites sur le terrain ainsi que les résultats des analyses multivariées et l'analyse discriminante canonique montrent l'existence de trois populations bovines locales sans bosse au Togo qui sont du Sud vers le Nord : la population bovine des Hybrides confirmés ou métis ou produits de divers métissages, la population bovine de Kouka et la population bovine Somba (Adanléhoussi et al., 2003 ; Moazami-Goudarzi et al., 2001). Dans chaque zone éco-floristique et pour chaque population bovine identifiée, les animaux sont connus également par un nom local qui renvoie aux tribus qui les élèvent. Sur la base des caractéristiques de cornage, le clivage est net entre Somba et Kouka d'une part et entre Somba et les hybrides confirmés d'autre part. En rapport avec les performances biométriques relevées, l'étude a montré un gradient décroissant des mesures (la longueur des oreilles, la longueur des cornes et la hauteur au garrot notamment) du Sud vers le Nord. En revanche, il a été souvent difficile de trancher entre le bovin Somba et la population bovine de Kouka, voire entre ce dernier groupe et celui appelé localement bovin Tém, dans la zone Tchaoudjo (Sokodé), au Sud de l'Atakora. Les éleveurs et les documents historiques (Lucien-Brun, 1987) sur l'origine et l'émigration des tribus de ces éleveurs au Togo ont rendu la tâche un peu plus facile.

La population bovine de l'Atakora sud proviendrait en majorité des sites d'élevage de Kouka. Aussi, le peuplement de la zone sud Atakora, tenu par les tribus «Tém » est récent (Lucien-Brun, 1987). La zone Nord-Ouest de l'Atakora tenue par les tribus d'éleveurs de Kouka (émigrés du Voltaïque vers le $17^{\text {ème }}$ siècle) est située sur la voie d'émigration du bovin Somba vers l'Ouest. Dans ce contexte, l'hypothèse plaide en faveur de l'unicité des bovins de Kouka et les bovins Somba. En revanche, la question subsidiaire reste encore les écarts statistiquement significatifs des mesures biométriques révélées par les tests multivariés et la distance de Mahalanobis calculée qui corroborent en partie les témoignages des éleveurs et les rapports
(ITRA, 2005 ; Adomefa et al., 2003). A cet effet, les animaux de Kouka sont des bovins Somba à la différence que les premiers présentent un format un peu plus grand. Les enquêtes antérieures (Adanléhoussi et al., 2003) dans la zone de Sokodé (Tchaoudjo) sur les bovins «Somba» rapportent des valeurs de moyennes de $116 \mathrm{~cm}$ pour $\mathrm{HG}$ et $137 \mathrm{~cm}$ pour le TP. Ces moyennes se rapprochent de celles enregistrées dans la présente étude. Cependant, pour ces mesures, Adanléhoussi et al. (2003) et Soro et al. (2015) ont trouvé des moyennes statistiquement inférieures, respectivement, sur le site naturel de la race Somba et les sites d'élevages de la race Baoulé en Côte d'Ivoire. Les résultats de la présente étude plaident ainsi en faveur d'un écotype (population bovine de Kouka) plus ou moins stabilisé qui serait issu d'un croisement retour entre la race Somba et la race Borgou. En effet, bien que la zone d'extension des bovins de Kouka coïncide d'après les études précédentes avec celle de la race Somba, dans cette zone, les croisements avec d'autres types, notamment avec la race Borgou, ont été nombreux (Kassa et al., 2016).

La prédominance du cornage en coupe et surtout la présence des cornes en Lyre caractéristiques des zébus dans les zones des bovins hybrides ou métis peuvent évoquer des croisements lointains ou récents avec les Zébus Peuhls transhumant ou les taurins à longues cornes comme les N'Dama.

Une limite de cette étude est la taille réduite de l'échantillon des mâles en raison de petits effectifs dans les troupeaux (certains troupeaux en sont même dépourvus) et surtout des moyens de contention rudimentaires utilisés au cours des mensurations.

La différentiation établie dans cette présente étude résulterait de l'effet des conditions agro-écologiques différentes associées aux pratiques (croisements et sélection) des éleveurs notamment pour l'amélioration du rendement en lait et viande (N'Goran et al., 2008). L'étude retient toutefois que les caractéristiques de cornages et les caractéristiques biométriques particulières plaident en faveur de trois 
populations de bovins d'un écotype « Kouka » différent, stabilisé ou peu stabilisé.

\section{Conclusion}

Cette étude a révélé l'existence de trois populations bovines locales sans bosse au Togo dont les caractéristiques morphobiométriques suivent un gradient décroissant du sud au nord du pays. Les informations collectées auprès des éleveurs ont également permis de définir trois sous-ensembles identifiés par un nom local. Ces résultats conduisent à rejeter l'hypothèse d'une homogénéité de la population locale bovine sans bosse au nord du Togo et à évoquer les facteurs environnementaux associés aux pratiques de production des éleveurs qui seraient à l'origine de différents types. Les résultats de la présente étude doivent être confirmés par une caractérisation moléculaire pour mieux évaluer la variabilité et la structure génétiques des populations bovines $\mathrm{du}$ Togo et confirmer l'existence de différentes «races» ou «d'écotypes» distincts.

\section{CONFLIT D'INTERETS}

Les auteurs déclarent qu'il n'existe aucun conflit d'intérêts lié à la présente étude.

\section{CONTRIBUTIONS DES AUTEURS}

SB a été l'investigateur principal du projet scientifique de ce présent manuscrit, dans la mise en œuvre du projet, il a réalisé la conception et la mise au point du protocole scientifique et des fiches d'enquête. Il a par la suite organisé et participé à la collecte des données sur le terrain. Il a élaboré également le protocole d'analyse statistique des données. L'interprétation des résultats et la rédaction du manuscrit ont été faites avec la contribution des autres auteurs. YN a supervisé les activités de ce travail. Il a contribué à la rédaction et à la révision critique du document. Il a également approuvé la version finale du présent manuscrit. G-KD a participé à la supervision des activités de ce travail. Il a participé à la rédaction et au recadrage des objectifs. Il a participé également à l'harmonisation du protocole, la collecte des données, l'interprétation scientifique des résultats et à la révision critique du manuscrit. Il a approuvé la présente version du présent manuscrit. BB a assuré la coordination scientifique et la planification des activités du projet. Il a contribué à l'interprétation scientifique et à la révision de la présente version du manuscrit. Il a approuvé la présente version du présent manuscrit. TN' a participé à la collecte des données sur le terrain et à la rédaction et à la révision du manuscrit. Il a approuvé la présente version du présent manuscrit.

\section{REMERCIEMENTS}

L'étude a été conduite grâce à la collaboration entre l'Institut Togolais de Recherche Agronomique et le Département de Zoologie de l'Université de Lomé avec l'appui financier du Programme de Productivité Agricole en Afrique de l'Ouest (PPAAO). Nous remercions également les chefs des communautés Peuhl et tous les éleveurs du «bovin local »du Togo.

\section{REFERENCES}

Adanléhoussi A, Bassowa H, Défly A, Djabakou K, Adoméfa K, Kouagou NT. 2003. Les performances de la race taurine Somba en milieu paysan. Tropicultura, 21(3): 135-141. DOI : http://www.tropicultura.org/text/v21n3/1 35 pdf.

Adomefa K, Bonfoh B. 2003. État des ressources génétiques animales au Togo. Rapport national : Lomé Mars 2003, p. 81.

Chabi China TF, Gbangboche AB, Attindehou S, DokoAllou S-Y, Salifou S, Pangui LJ, Abiola AF. 2016. Élevage de bovins Somba et gestion de son écologie parasitaire gastro-intestinal au Bénin. Int J. Biol. Chem. Sci., 10(5): 2300-2315. DOI:

http://dx.doi.org/10.4314/ijbcs.v10i5.28.

ITRA. 2005. Productions animales au Togo : Situation de référence. Rapport national. 128 pages.

Kassa SK, Ahounou S, Dayo G-K, Salifou C, Issifou, TM, Dotche I, Gandonou SP, 
Yapi-Gnaoré V, Koutinhouin GA M, Yousao AKI. 2016. Performances de production laitière des races bovines de l'Afrique de l'Ouest, Int. J. Biol. Chem. Sci., 10(5): 2316-2330. DIO : http://dx.doi.org/10.4314/ijbcs.v10i5.29.

Lombo Y. 2002. Cartographie des races bovines dans la zone du CIRDES. Mémoire d'Ingénieur des Travaux d'élevage, Université de Lomé, Lomé, $137 \mathrm{p}$.

Lucien-Brun B. 1987. Migration et colonisation des terres neuves. In Migrations Rurales des Kabyè et des Losso. Collection: Travaux et Documents ORSTOM : Paris ; 1-215.

Moazami-Goudarzi K, Belemsaga DMA, Ceriotti G, Laloë D, Fagbohoun F, Kouagou N, Sidibé I, Codjia V, Crimella MC, Grosclaude F, Touré SM. 2001. Caractérisation de la race bovine Somba à l'aide de marqueurs moléculaires. Revue Élev. Méd. vét. Pays Trop., 54(2): 129-138. DOI : https://agritrop.cirad.fr/489759/1/ID4897 59.
N'goran KE, Yapi-Gnaoré CV, Fantodji A, Ahoussou N. 2008. Caractérisation phénotypique et performances zootechniques des vaches laitières de trois régions de Côte d'Ivoire, Archivos de Zootecnia ,57(220): 415-426.

Soro B, Sokouri PD, Dayo G-K, N'Guetta ASP, Yapi-Gnaoré CV. 2015. Morphometric and physical characteristics of Baoulé cattle in the "Pays Lobi" of Côte d'Ivoire. LRRD, 27(7): $\quad$ 0121-3784. DOI: http://www.lrrd.org/lrrd27/7/soko27124. html.

Talaki E, Dao B, Dayo G-K, Alfa E, N'Féidé T. 2014. Trypanosomoses animales dans la Plaine de Mô au Togo, Int. J. Biol. Chem. Sci., 8(6): 2462-2469. DOI : http://dx.doi.org/10.4314/ijbcs.v8i6.9.

Youssao AKI, Dahouda M, Attakpa EY, Koutinhouin GB, Ahonou GS, Toleba SS, Balogoun BS. 2013. Diversité des systèmes d'élevages de bovins de race bovine Borgou dans la zone soudanienne du Bénin, Int. J. Biol. Chem. Sci., 7(1): 125-146. DOI : http://dx.doi.org/10. 4314/ ijbcs.v7i1i.11. 